(c) American Dairy Science Association, 2006.

\title{
Two Mathematical Programming Models of Cheese Manufacture
}

\author{
J. A. Burke \\ Department of Agricultural and Applied Economics, University of Wisconsin, Madison 53706
}

\begin{abstract}
The standardization problem faced by cheese makers is formulated as a nonlinear programming problem using the assumptions of the Van Slyke cheese yield formula. The objective function of the model is to minimize the net cost of producing a given quantity of cheese subject to a set of production constraints. An approximation of the standardization problem formulated as a linear programming problem is also presented. Two different approaches to finding a solution are provided. The model is implemented in Microsoft Excel and solved with the standard add-in solver available in that program. An example is provided to contrast the difference between the nonlinear programming and its linear approximation, and a second example is used to illustrate the yield implications of ultrafiltered milk protein products in Cheddar cheese production. Additionally, a method for pricing inputs using the sensitivity analysis generated by the solver is demonstrated.
\end{abstract}

Key words: cheese, optimization, mathematical programming, milk protein concentrate

\section{INTRODUCTION}

Mathematical programming models, also referred to as optimization models, are powerful tools for use in both industry and science. The benefits to industry are clear, as these models are designed to represent the decisions faced by executives and to show the profitmaximal or cost-minimal strategies to pursue. For the scientist, a well-formulated problem captures the variables and constraints essential to a process, allowing these models to act as common points of reference to researchers in the same field, as well as a framework in which to consider new avenues of inquiry.

One of the earliest mathematical programming models of cheese making was a linear program $(\mathbf{L P})$ developed by Kerrigan and Norback (1986). Their model had 4 essential constraints plus any optional resource availability constraints. The cheese milk could be standard-

Received September 14, 2005.

Accepted October 11, 2005.

E-mail: josephburke@wisc.edu ized in the model by skimming cream or with the addition of any of 4 inputs: raw milk, NDM, skim milk, and condensed milk.

More recently, Papadatos et al. (2002) published a nonlinear programming (NLP) model of cheese manufacture. This model used 42 constraints to describe the cheese-making process. The Papadatos et al. (2002) model allowed for an arbitrary number of possible standardization methods, including the removal of cream or the addition of raw milk, ultra- or microfiltered milk, or NDM. This model was later extended by Papadatos et al. (2003) to evaluate the economic feasibility of microfiltration prior to cheese making. Their study used the same description of cheese manufacturing as the 2002 study, but the new model included an additional set of constraints to describe the microfiltration process, whereby some of the available milk is first microfiltered before it is added in the cheese milk.

In addition to the Kerrigan and Norback (1986) and the Papadatos et al. $(2002,2003)$ models, a third model of cheese manufacture was developed by Barcenas in her dissertation (Barcenas, 1999). The Barcenas model is a LP model, and it considers a large set of potential inputs, including ultrafiltered milk. Barcenas expressed the constraints on cheese manufacture on a component basis, and consequentially, her model is very similar to the model presented here, although not identical.

Although cheese manufacturers are assumed to maximize profit, their problem could be described as a net cost-minimization problem for a number of reasons. First, a manufacturer who maximizes profit automatically acts to minimize his production costs at the optimal production level, solving the problem described here as a subproblem within his profit-maximization problem. In the profit-maximization problem, the manufacturer chooses the level of inputs to be supplied and outputs produced to maximize his profit. This problem may be separated into 2 stages: the manufacturer first chooses his inputs to minimize his net costs for every possible production level and then chooses the production level that maximizes his profit. The net cost-minimization problem is, therefore, the first stage in this 2stage profit-maximization problem. This is a standard result from the economic theory (Silberberg and Suen, 2001). 
Second, if the solution to the net cost-minimization problem is scalable in the inputs and outputs, the profitmaximization problem can only be solved by assuming additional structure in the model. The models presented here possess this scaling property: increasing quantity of cheese to be produced by a factor increases the optimal quantity of cheese milk and input levels by that same factor. Net costs also increase by the same factor, as do profits under the ordinary assumption of a constant output price. If the cheese maker makes $\$ 1$ on $10 \mathrm{lbs}$ of cheese, he will make $\$ 100$ on $1,000 \mathrm{lbs}$ of cheese, and $\$ 1$ million on 10 million lbs of cheese. The scale of production variable is unbounded, so the optimal quantity of cheese to produce is infinite. The net cost-minimization problem, however, has a solution at every possible scale of production, i.e., when the scale of production variable is held constant. Thus, the net cost problem may be solved even when the profit-maximization problem cannot.

Third, there are situations when the cost-minimal solution is more useful than a profit-maximization solution. Such situations occur when the price associated with the cheese produced may be difficult to derive or when the production level is fixed. The output price is difficult to derive, for instance, if a cheese maker wants to make a cheese for his personal consumption. The cheese is not sold, but he would still want to minimize the cost of producing the desired cheese. The cost-minimizing formulation of the problem may also be more useful when cheese is packaged jointly with other products, such as crackers. Here, there is no explicit price associated with the cheese, only the price of the cheese and crackers together. Cost-minimal solutions are also more useful than profit-maximal solutions when the production level is fixed by contract. In this case, the only flexibility the manufacturer has is in his cost decisions. In all of these examples, the formulation of the profit-maximization problem is either difficult to formulate or irrelevant, whereas the application of the net cost-minimization model to cheese production is still appropriate and straightforward.

In the next section, a conceptual model of dairy products is presented. The conceptual model is used to describe the inputs and outputs, but it may be used to describe any dairy product, including inputs not considered here. The third section develops a nonlinear model of the net cost minimization problem faced by the cheese manufacturer, as well as its linear approximation. This section also describes and gives justification for the constraints in the 2 models. The fourth section provides a solution algorithm for the nonlinear model. Two examples are given in the fifth section, one comparing results from the linear and nonlinear models and a second showing the yield implications of using ultrafiltered milk and milk protein concentrate in cheese production.

\section{MATERIALS AND METHODS}

In this section, I begin by developing a conceptual model of dairy products. Using this model, I then describe the constraints and objectives faced by cheese makers. Adding the objective to the constraints forms the model of cheese making. The model is technically a NLP problem, but it has a closely related linear approximation. I describe 2 methods of solving the nonlinear problem, which I implement in Excel (Microsoft, Seattle, WA) using the Solver add-in (Frontline Systems, Inc., Incline Village, NV) for that program.

\section{Conceptual Model of Dairy Products}

Throughout this paper, the letter $k$ denotes a dairy product, and the set of dairy products is $K$. This set is arbitrary and varies from application to application. Dairy products considered here are cheese (che), whole milk $(w m)$, skim milk $(\mathrm{sm})$, cream $(\mathrm{crm})$, NDM, milk protein concentrate $(\mathrm{mpc}$ ), and on-site ultrafiltered milk (ufos). Thus, the set of dairy products is

$$
K \equiv\{c h e, w m, s m, c r m, \mathrm{NDM}, m p c, u f o s\} .
$$

Therefore, the letter $k$ is a generic element of $K$. Onsite ultrafiltered milk and milk protein concentrate are together referred to as "ultrafiltered milk protein products."

The conceptual model of dairy products used here is to identify each product by 6 mutually exclusive and exhaustive components: fat ( fat), casein (cas), whey protein (whe), lactose (lac), other solids (oth), and water (wat). In addition to these, I also make reference to the serum (ser), solids (sol), and serum solids (sersol) content of dairy products. The solids portion is defined as everything but the water, and the serum portion consists of the whey protein, lactose, other solids, and water. Serum solids are the solids portion of the serum: whey protein, lactose, and other solids. The set of all components is $L$, where $l$ a generic element of $L$. From these definitions,

$$
L \equiv\{f a t, \text { cas, whe, lac, oth, wat, ser, sol, sersol }\}
$$

The set of mutually exclusive and exhaustive components is

$$
\bar{L} \equiv\{f a t, c a s, \text { whe, lac, oth, wat }\} .
$$

Let $\lambda_{k, l}$ be the percentage of $l$ in product $k$, expressed as a ratio. Because the set $\bar{L}$ is exhaustive, it is true that 
Table 1. Composition assumptions

\begin{tabular}{lrrrrr}
\hline & Fat & Casein & $\begin{array}{l}\text { Whey } \\
\text { protein }\end{array}$ & Lactose & $\begin{array}{r}\text { Other } \\
\text { solids }\end{array}$ \\
\cline { 2 - 3 } & & & & & \\
Whole milk & 3.700 & 2.620 & 0.700 & 4.700 & 0.800 \\
Skim milk & 0.050 & 2.730 & 0.730 & 4.890 & 0.830 \\
Cream & 40.000 & 1.530 & 0.410 & 2.740 & 0.180 \\
Nonfat dry milk & 0.500 & 28.500 & 7.600 & 51.000 & 8.700 \\
Milk protein concentrate & 1.000 & 55.600 & 14.900 & 19.900 & 8.500 \\
On-site ultrafiltered milk & 0.300 & 15.300 & 4.100 & 7.500 & 2.500 \\
Cheese & 32.700 & 23.900 & n/a 1 & n/a & n/a \\
\hline
\end{tabular}

${ }^{1} \mathrm{n} / \mathrm{a}=$ not applicable.

for all $k$ in $K, \sum_{l \in \bar{L}} \lambda_{k, l}=1$.

This equation states that, for each product, the sum of the percentages of the exhaustive components for that product should account for $100 \%$ of the composition of the product. From the definition of the solids composition of dairy products, it is also true that

$$
\begin{gathered}
\text { for all } k \text { in } K, \lambda_{k, \text { sol }}= \\
\lambda_{k, f a t}+\lambda_{k, \text { cas }}+\lambda_{k, \text { whe }}+\lambda_{k, \text { lac }}+\lambda_{k, \text { oth }}=1-\lambda_{k, \text { wat }} .
\end{gathered}
$$

The definition of the serum implies that

for all $k$ in $K, \lambda_{k, \text { ser }}=\lambda_{k, w h e}+\lambda_{k, l a c}+\lambda_{k, o t h}+\lambda_{k, w a t}$.

Similarly, for serum solids it is true that

$$
\text { for all } k \text { in } K, \lambda_{k, \text { sersol }}=\lambda_{k, w h e}+\lambda_{k, \text { lac }}+\lambda_{k, \text { oth }} \text {. }
$$

Table 1 provides the composition assumptions used in the model. The cheese composition is similar to that of Cheddar cheese. The milk protein concentrate used in this model is MPC 70.

Let $K_{x}$ be the set of products used as inputs in cheese production,

$$
K_{x} \equiv\{w m, s m, c r m, \mathrm{NDM}, m p c, u f o s\} .
$$

[For legibility, $K_{x}$ is written $K(x)$ in subscripts.] For each $k$ in $K$, the variable $x_{k}$ is the amount of that product used in cheese production. Additionally, let $y$ be the amount of cream skimmed and $z$ be the amount of cheese to be produced. Also note that cream is both an input and an output, as it may be either added to or skimmed from the cheese milk. Finally, the whey stream is also an output from the cheese-making process. This model does not consider the processing of the whey stream, but values the whey stream on a component basis. Accordingly, for each component $l$ in $L$, the corresponding variable $w_{l}$ is the amount of component $l$ in the whey stream.

\section{Models of Cheese Manufacture}

Let $X$ be the mass of the cheese milk, calculated as the sum of the masses of the inputs less the mass of skimmed cream:

$$
\sum_{k \in K(x)} x_{k}-y=X
$$

For all $l, X_{l}$ will denote the quantity of component $l$ in the cheese milk. [For legibility, $X_{l}$ is written $X(l)$ in subscripts.] These are calculated as the sum, by component, of all inputs less skimmed cream $(y)$ :

$$
\text { for all } l, \sum_{k \in K(x)} x_{k} \lambda_{k, l}-y \lambda_{c r m, l}=X_{l} \text {. }
$$

For $l$ in the set $\{f a t$, cas $\}$, let $\eta_{l}$ be the corresponding retention factor. Let $\sigma$ be the salt solids retention factor. The cheese yield is denoted in the same units as the $X_{l}$ variables. Here, the units are assumed to be kilograms. The Van Slyke cheese yield formula is

$$
z=\frac{\left(X_{f a t} \eta_{f a t}+X_{c a s} \eta_{c a s}\right) \times \sigma}{1-\lambda_{\text {che, wat }}} .
$$

The numerator of this equation is the amount (in $\mathrm{kg}$ ) of solids retained in the cheese from the cheese milk, and the denominator is the percent solids in each $\mathrm{kg}$ of cheese.

Of note is the salt solids retention factor, $\sigma$, which enters the equation differently from the fat and casein retention factors. The salt solids retention factor reflects added salt plus the serum solids portion of the cheese milk. The Van Slyke formula incorporates the assumption that the amount of serum solids retained in the cheese is in fixed proportion to the fat and casein in the cheese milk.

When the amount of cheese produced is fixed at $z$, the assumptions underlying the Van Slyke formula imply an exact amount of fat and casein in the cheese 
milk. The fat retained from the cheese milk must be equal to the fat in the cheese:

$$
X_{\text {fat }} \eta_{\text {fat }}=z \lambda_{\text {che,fat }} .
$$

The left-hand side is the amount of fat in the cheese milk multiplied by the fat retention factor. The righthand side of this equation is the amount of cheese to be manufactured multiplied by the amount of fat in each pound of cheese. Similarly, the casein retained from the cheese milk must equal the casein in the cheese:

$$
X_{\text {cas }} \eta_{\text {cas }}=z \lambda_{\text {che,cas }} \text {. }
$$

Equations 4 and 5 are the fat and casein requirements of the cheese milk, respectively. If these requirements are satisfied, a set of retention factors and a fixed ratio of $\lambda_{\text {che,cas }}$ to $\lambda_{\text {che,fat }}$ imply a fixed ratio of casein to fat in the cheese milk:

$$
\frac{X_{c a s}}{X_{f a t}}=\frac{\eta_{f a t}}{\eta_{c a s}} \frac{\lambda_{c h e, c a s}}{\lambda_{c h e, f a t}} .
$$

Through this relationship, the levels of casein and fat required in the cheese milk are controlled through the cheese composition parameters and fat and casein retention factors. The composition of the cheese used in the model is determined by the type of cheese to be manufactured, and the retention factors are determined by the intended cheese, the method by which it is produced, and the skill of the manufacturer.

The common assumption used by cheese makers is that the serum solids composition of the cheese is identical to the serum solids composition of the cheese milk from which it was produced. This assumption means that the serum solids composition of the cheese varies depending on the method of standardization of the cheese milk. To distinguish the serum solids composition of the cheese from the other fixed compositions in the model, the variable compositions will be identified by $\tilde{\lambda}_{c h e, l}$. In each kilogram of cheese, the amount of each serum solid component per kilogram is

$$
\begin{gathered}
\text { for all } l \text { in }\{w h e, l a c, o t h\}, \\
\tilde{\lambda}_{\text {che }, l}=\lambda_{\text {che,sersol }} \frac{X_{l}}{X_{\text {sersol }}}
\end{gathered}
$$

Cheese makers face other constraints in addition to the fat and casein requirements of the cheese milk. The most fundamental of these is the set of mass balance constraints. These constraints occur in every production process and simply state that for each component, the amount of a component in the inputs must equal the amount of that same component in the outputs. Using the established notation, this set of constraints may be expressed as

$$
\begin{gathered}
\text { for all } l \text { in }\{\text { fat }, \text { cas }\}, \\
\sum_{k \in K(x)} \lambda_{k, l} x_{k}=z \lambda_{\text {che }, l}+y \lambda_{\text {crm }, l}+w_{l}, \\
\text { for all } l \text { in }\{w h e, l a c, \text { oth }\}, \\
\sum_{k \in K(x)} \lambda_{k, l} x_{k}=z \tilde{\lambda}_{\text {che }, l}+y \lambda_{\text {crm }, l}+w_{l} .
\end{gathered}
$$

The equation for water is not included in Equation 8 , as its inclusion makes the system of equations formed by Equations 2 and 8 linearly dependent. Substituting Equation 7 into 8 shows that the mass balance equations for the serum components are nonlinear, as $X_{\text {sersol }}$ appears in the denominator. These equations are also not convex. The nonlinear equations, Equations 7 and 8 , may be made linear and convex, however, by replacing the variable $X_{\text {sersol }}$ with a parameter that approximates it, $\hat{X}_{\text {sersol }}$. The parameter $z$ is already held constant in the problem. If $\hat{X}_{\text {sersol }}$ is also constant, then all of the mass balance equations are linear, including those for the serum components. However, substituting the approximate $\hat{X}_{\text {sersol }}$ for the exact $X_{\text {sersol }}$ implies that the serum components in the whey stream will also be only approximations. Thus, for $l$ in the set \{whe, lac, oth $\}, w_{l}$ is replaced with $\hat{w}_{l}$. The exact mass balance equations may now be approximated as

$$
\begin{gathered}
\text { for all } l \text { in }\{\text { fat, cas }\}, \\
\sum_{k \in K(x)} \lambda_{k, l} x_{k}=z \lambda_{\text {che }, l}+y \lambda_{\text {crm }, l}+w_{l}, \\
\text { for all } l \text { in }\{w h e, \text { lac, oth }\}, \\
\sum_{k \in K(x)} \lambda_{k, l} x_{k}=z \lambda_{\text {che,sersol }} \frac{X_{l}}{\hat{X}_{\text {sersol }}}+y \lambda_{\text {crm }, l}+\hat{w}_{l} .
\end{gathered}
$$

Note that the Equations 8 and 9 are equal when $X_{\text {sersol }}=\hat{X}_{\text {sersol }}$.

Cheese makers also face a limit on the amount of lactose in the cheese. Ordinarily, this requirement is stated as a limit on the amount of lactose in the cheese milk. The common rule of thumb is that the lactose content of the cheese milk must be $<5 \%$ of the cheese milk. Implicitly, this constraint assumes that the serum in the cheese milk is fixed. Under this assumption, a limit on the cheese milk results in a limit on the lactose in the cheese according to Equation 7. I suggest that this statement should be restated as a constraint on 
the lactose in the resulting cheese, instead of the cheese milk. Let $\mu_{\text {che, lac }}$ be the upper limit on the lactose in the cheese. From Equation 7, this implies

$$
\lambda_{\text {che,sersol }} \frac{X_{\text {lac }}}{X_{\text {sersol }}} \leq \mu_{\text {che,lac }},
$$

which may be rearranged as a linear constraint,

$$
\lambda_{\text {che,sersol }} X_{\text {lac }} \leq \mu_{\text {che,lac }} X_{\text {sersol }} .
$$

Cheese makers are also constrained by a limit on the viscosity of the cheese milk. Cheese milk is stirred by a machine in cheese manufacturing, but it cannot be stirred when the cheese milk is too thick with solids. This viscosity constraint is ordinarily expressed as an upper limit on the amount of solids in the cheese milk. Let $\mu_{X, s o l}$ be the upper limit on the solids in the cheese milk. The constraint is

$$
\frac{X_{s o l}}{X} \leq \mu_{X, s o l},
$$

which may be expressed as a linear constraint by multiplying through by $X$,

$$
X_{s o l} \leq \mu_{X, s o l} X .
$$

The variable $X$ is the mass of the cheese milk, and $X_{\text {sol }}$ is the mass of solids in the cheese milk. This constraint could be replaced by similar limits on the amount of fat and casein in the cheese milk.

Cheese manufacturers may also be constrained by input availability. For all $k$ in $K_{x}$, let $\mu_{k}$ be the amount of $x_{k}$ that is available for use in cheese manufacture. Any input availability constraints have the form

$$
\text { for all } k \text { in } K_{x}, x_{k} \leq \mu_{k} \text {. }
$$

This equation simply states that the amount of $x_{k}$ used in cheese manufacture cannot exceed what is available.

Cream may be added to or removed from the cheese milk, activities represented by the variables $x_{c r m}$ and $y$, respectively. The two variables have different associated costs in the objective function, $c_{c r m}$ and $p_{c r m}$, also, respectively. If $p_{c r m}>c_{c r m}$, then the cheese maker may sell cream at a price higher than he purchases it and could potentially make an infinite amount of money by adding and removing an infinite amount of cream. The solution would also be unbounded if the cheese maker could acquire cream indirectly, such as by skimming it from whole milk, if the implicit price of cream in the whole milk was less than the price at which he could sell it, and if the supply of cream acquired in this manner is not bounded by other constraints. To prevent this, the amount of cream that may be removed is set to an arbitrary upper limit that is proportional to the amount of cheese to be produced. Let $\phi$ be the upper limit on the cream that may be removed as a proportion of the cheese. The constraint is written as

$$
y \leq \varphi z .
$$

The objective function in the model is to minimize the net cost of producing the given quantity of cheese, $z$. The net cost is the net input cost plus any costs associated with processing the cheese milk. Let $C$ be the net cost function, $c_{k}$ be the price associated with input $x_{k}$, let $p_{\text {crm }}$ be the price per pound of skimmed cream, and let $r_{l}$ be the value of whey stream component $w_{l}$. The net input cost function is

$$
\mathrm{C}_{0}(c, p, r, x, y, w)=\sum_{k \in K(x)} c_{k} x_{k}-p_{c r m} y-\sum_{l \in \bar{L}} r_{l} w_{l} .
$$

The net input cost is the cost per unit of the inputs, $x_{k}$ less any revenue generated by the skimmed cream, $y$, or from the whey stream, $w_{l}$. For simplicity, this function is usually written as $C_{0}(x, y, w)$, where the dependence of costs on the constant price parameters $c$, $p$, and $r$ is implicit. This particular formulation assumes the whey stream components are known exactly in the problem. If they are only known approximately, then $\hat{w}_{l}$ substitutes for $w_{l}$ as appropriate.

The net input cost function is independent of cheese yield. To see this, assume two different methods of standardization may have the same net input cost but different cheese yields. Intuitively, one would expect a manufacturer faced with two such possibilities to choose the method with the higher cheese yield. This is a tradeoff between yields and costs not captured in the net input cost function. Let $\theta$ be the yield, defined as the amount of cheese produced, $z$, per pound of cheese milk, $X$,

$$
\theta \equiv \frac{z}{X}
$$

Because $X$ is a variable, $\theta$ is a variable. This formula indicates that the trade-off between yield and costs can be captured through costs associated with the cheese milk variable $X$. I will here refer to costs associated with the quantity of cheese milk as vat costs, as the amount of cheese milk used to produce cheese is equal to the amount of vat capacity used. Let $v$ be the vat costs. Vat costs are incorporated into the net input cost function to form the net cost function, 
Table 2. Parameter assumptions and prices

\begin{tabular}{lll}
\hline Parameter & Symbol & Value \\
\hline Fat retention factor & $\eta(\mathrm{fat})$ & 0.93 \\
Casein retention factor & $\eta(\mathrm{cas})$ & 0.96 \\
Ratio of mass of serum solids to mass of Cheddar cheese & $\lambda(\mathrm{che}, \mathrm{sersol})$ & 0.029 \\
Upper limit on ratio of lactose to serum solids in cheese & $\mu(\mathrm{che}, \mathrm{lac})$ & 0.80 \\
Upper limit on ratio of solids in cheese milk to mass of cheese milk & $\mu(\mathrm{X}, \mathrm{sol})$ & 0.15 \\
Upper limit on ratio of cream removal to cheese production & $\varphi$ & 1.00 \\
Vat cost (/kg of cheese milk) & $\mathrm{v}$ & $\$ 0.037$ \\
Commodity & & Price $(\$ / \mathrm{kg})$ \\
\hline Cost of whole milk & $\mathrm{c}(\mathrm{wm})$ & $\$ 0.238$ \\
Cost of skim milk & $\mathrm{c}(\mathrm{sm})$ & $\$ 0.159$ \\
Cost of added cream & $\mathrm{c}(\mathrm{crm})$ & $\$ 1.140$ \\
Cost of nonfat dry milk & $\mathrm{c}(\mathrm{nfdm})$ & $\$ 1.985$ \\
Cost of milk protein concentrate & $\mathrm{c}(\mathrm{mpc})$ & $\$ 3.616$ \\
Cost of on-site ultrafiltered milk & $\mathrm{c}(\mathrm{ufos})$ & $\$ 0.992$ \\
Price of skimmed cream & $\mathrm{r}$ & $\$ 1.118$ \\
Price of fat in whey stream & $\mathrm{r}(\mathrm{fat})$ & $\$ 2.360$ \\
Price of casein in whey stream & $\mathrm{r}(\mathrm{cas})$ & $\$ 5.386$ \\
Price of whey protein in whey stream & $\mathrm{r}(\mathrm{whe})$ & $\$ 1.476$ \\
Price of lactose in whey stream & $\mathrm{r}(\mathrm{lac})$ & $\$ 0$ \\
Price of other solids in whey stream & $\mathrm{r}(\mathrm{oth})$ & $\$ 0$ \\
Price of water in whey stream & $\mathrm{r}(\mathrm{wtr})$ & $\$ 0$ \\
\hline
\end{tabular}

$$
\begin{gathered}
C(x, y, w, X)= \\
\sum_{k \in K(x)} c_{k} x_{k}-p_{c r m} y-\sum_{l \in \bar{L}} r_{l} w_{l}+v X .
\end{gathered}
$$

Now 2 different methods of standardization may have the same net input $\operatorname{cost} C_{0}$, but if one method has a higher yield, then it will also have a lower net cost function $C$. Also, as before, if the whey stream components are only known approximately in the problem, then $\hat{w}_{l}$ substitutes for $w_{l}$ as appropriate. The net cost function is the objective function to be minimized in the optimization problem.

Table 2 provides all of the parameter assumptions used in the model. The fat and casein retention factors are those for Cheddar cheese. The upper limit on the lactose to serum solids ratio, 0.80 , corresponds roughly to a 5\% upper limit on lactose in the cheese milk under ordinary standardization techniques. The limit on the amount of solids in the cheese milk is 0.15 or $15 \%$. The vat cost is $\$ 0.037 / \mathrm{kg}$ of cheese milk, which is calibrated to the opportunity cost of unused capacity. This is approximately equal to the average yield per kilogram of cheese milk multiplied by the profit per kilogram of cheese. Assumptions used in calculating the vat cost are a yield of 0.10 and profits of $\$ 0.37 / \mathrm{kg}$ of cheese. The prices roughly correspond to 2002 prices from USDA. The milk protein price is the import price for 2002 reported by the U.S. International Trade Commission, and the on-site ultrafiltered milk price is an estimate.

The cheese maker's objective is to minimize the net cost of producing a given quantity of cheese. He is con- strained by the fat and casein requirements of the cheese, mass balance constraints, limits on the amount of lactose in the cheese and the solids in the cheese milk, and an arbitrary upper limit on the amount of cream that may be removed. Additionally, all variables are assumed to be nonnegative. This problem may be written mathematically as

$$
\min _{w, x, y, X, X(l)} C(x, y, w, X)
$$

subject to Equations 1, 2, 4, 5, 7, 8, 11, 13, 14, and 15,

$$
\begin{gathered}
x \geq 0, y \geq 0, X \geq 0, \text { and for all } l \text { in } L, \\
w_{l} \geq 0 \text { and } X_{l} \geq 0 .
\end{gathered}
$$

This set of equations constitutes the exact problem faced by the cheese maker, and it is nonlinear because of Equation 7. Thus, this is a NLP problem.

As stated earlier, the nonlinearity in the mass balance equations is removed if the cheese maker chooses to work with an approximate value of the serum solids, $\hat{\mathrm{X}}_{\text {sersol }}$, and approximate values of the whey protein, lactose, and other solids in the whey stream, $w_{l}$. By using these values, the cheese maker solves a problem that approximates the exact problem. Let

$$
\begin{gathered}
\hat{C}(w, \hat{w}, x, y, X)=\sum_{k \in K(x)} c_{k} x_{k}-p_{\mathrm{crm}} y \\
-\sum_{l \in(f a t, c a s, w a t)} r_{l} w_{l}-\sum_{l \in(w h e, l a c, o t h)} r_{l} \hat{w}_{l}-v X .
\end{gathered}
$$


Here, the variable $w$ refers only to the whey stream components $l$ in $\{$ fat, cas, wat $\}$, and $w$ refers to those components $l$ in $\{w h e, l a c, o t h\}$. The approximate cheese maker problem is expressed mathematically as

$$
\min _{w, \hat{w}, x, y, X, X(l)} \hat{C}(w, \hat{w}, x, y, X)
$$

subject to Equations 1, 2, 4, 5, 9, 11, 13, 14, and 15 .

$$
x \geq 0, y \geq 0 \text {, and for all } l \text { in } L, w_{l} \geq 0 \text { and } X_{l} \geq 0 .
$$

This approximate problem is a LP problem. I refer to this problem as the approximate LP problem, and the constraints for this problem are the approximate $L P$ constraints. The only difference between the approximate LP and the NLP is the use of Equation 9 instead of Equations 7 and 8. Once the approximate LP is solved, the exact whey stream composition may be calculated from the solution by using Equation 8 and the serum identity equations.

\section{Solution Algorithm for the NLP}

With one additional constraint, the approximate LP problem may be used to solve the NLP problem. In the LP problem, the actual serum solids, $X_{\text {sersol }}$, will not, in general, equal the approximate serum solids, $X_{\text {sersol }}$. This inconsistency is resolved with the addition of the consistency constraint:

$$
X_{\text {sersol }}=\hat{X}_{\text {sersol }} \text {. }
$$

Incorporating this into the LP problem means that the serum solids in the cheese milk are now artificially constrained by the restriction that the assumption about the serum solids content of the cheese milk, $\hat{X}_{\text {sersol }}$, is always correct. The inclusion of the consistency constraint in the approximate LP forms the consistent LP problem, which is

$$
\min _{w, \hat{w}, x, y, X, X(l)} \hat{C}(w, \hat{w}, x, y, X)
$$

subject to the approximate LP constraints and $X_{\text {sersol }}=\hat{X}_{\text {sersol }}$ (consistency constraint).

Solving the consistent LP guarantees a solution where the assumption is consistent with the actual serum solids content, but this solution is not necessarily optimal, i.e., the minimum net cost solution to the nonlinear model. However, the consistent LP may be used in an algorithm to solve the nonlinear problem.

The serum solids content is a one-dimensional variable (not a vector), and it can only take on a limited range of values. To determine the lower bound of this range, a LP problem is formed by taking the constraints from the approximate LP and using the objective function (and choice variables),

$$
\min _{w, \hat{w}, x, y, X, X(l)} X_{\text {sersol }}
$$

subject to the approximate LP constraints.

The upper bound may be similarly determined using the same set of constraints and maximizing the objective function,

$$
\max _{w, \hat{w}, x, y, X, X(l)} X_{\text {sersol }}
$$

subject to the approximate LP constraints.

Let $\mu_{\text {sersol }}^{\text {low }}$ and $\mu_{\text {sersol }}^{u p}$ be the lower and upper bounds to the serum solids content of the cheese milk as determined from these $2 \mathrm{LP}$, respectively.

For each assumption on $\hat{X}_{\text {sersol }}$, there is an associated minimum net cost from the solution to the consistent LP problem. By letting the assumptions of $\hat{X}_{\text {sersol }}$ vary over the range and solving the consistent LP problem at each different value, an algorithm can solve the nonlinear problem by calculating the minimum of all solutions to the consistent LP problems over the range of possible assumptions on $\hat{X}_{\text {sersol }}$.

Let $C^{\prime}\left(\hat{X}_{\text {sersol }}\right)$ be the minimum net cost of the consistent LP for a particular assumption of $\hat{X}_{\text {sersol }}$, and let $C^{*}$ be the minimum net cost solution to the nonlinear problem. The algorithm used to solve the nonlinear problem is

Initialize $\hat{X}_{\text {sersol }}=\mu_{\text {sersol }}^{\text {low }}$.

Solve the consistent LP for $C^{\prime}\left(\hat{X}_{\text {sersol }}\right)$.

Initialize $X_{\text {sersol }}^{*}=\hat{X}_{\text {sersol }}$ and $C^{*}=C^{\prime}\left(\hat{X}_{\text {sersol }}\right)$.

Choose an increment by which to increase the serum solids content of the cheese milk. Let this increment be $\varepsilon$.

Increment $\hat{X}_{\text {sersol }}=\hat{X}_{\text {sersol }}+\varepsilon$.

If $\hat{X}_{\text {sersol }}>\mu_{\text {sersol }}^{u p}$, then exit.

Solve the consistent LP for $C^{\prime}\left(X_{\text {sersol }}\right)$. $\hat{X}_{\text {sersol. }}$.

If $C^{\prime}\left(X_{\text {sersol }}\right)<C^{*}$, then set $C^{*}=C^{\prime}\left(X_{\text {sersol }}\right)$ and $X_{\text {sersol }}^{*}=$

Return to increment step. Upon exit, the minimal $\operatorname{cost}$ is $C^{*}$, and the cost-minimizing serum solids content is $X_{\text {sersol }}^{*}$.

This algorithm works because all possible values for the serum solids variable, $X_{\text {sersol }}$, lie between its lower and upper bounds, and at each value of $\hat{X}_{\text {sersol }}$ there is a solution to the consistent LP. 


\section{Implementation}

The nonlinear model and its linear approximation may be implemented in any mathematical programming environment that allows for loop control. Here, the models were written and solved in Excel. Aside from the nonnegativity constraints, the constraints and the objective function are written out directly in the spreadsheet. A macro was written with Visual Basic (Microsoft) to solve the approximate LP using the default addin solver. The NLP is solved by iterating the consistent LP using the algorithm specified in the previous section. For both the approximate LP and the consistent LP, the default settings of the solver are used with the exception of the "Assume Linear Model" and "Assume Non-Negative" options, both of which are checked. The nonnegativity constraints do not need to be written out when the nonnegativity option is checked, and checking the linear model option means that the model is assumed to be linear and solved with the Simplex method. The NLP solution algorithm was implemented using a second Visual Basic macro. Both macros, for the approximate LP and the NLP, were associated with buttons on the spreadsheet.

\section{RESULTS AND DISCUSSION}

Two examples of applications of the model are discussed here. In the first example, milk protein concentrate and ultrafiltered milk are removed from the set of allowable inputs, and the approximate LP and NLP are both solved. The purpose of this example is to illustrate the relatively small differences between the 2 models. In the second example, the model is solved 3 times to illustrate the yield implications of using ultrafiltered milk protein products.

\section{Example 1}

In this example, I solve the model using, first, the approximate LP and then, using the NLP. The parameters are calibrated for Cheddar cheese, and the quantity of cheese to be produced, $z$, is $10 \mathrm{~kg}$. The exact whey stream is recovered from both solutions using the cheese milk identities and Equation 8. Use of ultrafiltered milk protein products is set to zero in this example, limiting the available inputs to whole milk, skim milk, cream, and NDM. This restricted set of inputs implies a range of feasible serum solids, $X_{\text {sersol }}$, between 5.857 and 5.891 in the cheese milk. The midpoint of the range is the serum solids composition assumed for the approximate LP, $\hat{X}_{\text {sersol }}=5.874$.

Table 3 provides the solution to the 2 models. The first column is the solution to the LP model using the
Table 3. A comparison of the LP (linear programming) and NLP (nonlinear programming) solutions

\begin{tabular}{lclc}
\hline & \multicolumn{3}{c}{$\begin{array}{l}\text { LP with exact } \\
\text { mass balance } \\
\text { equations }\end{array}$} \\
Variable & LP & NLP \\
\cline { 2 - 4 } Whole milk & 95.0 & 95.0 & \\
Skim milk & 0 & 0 & 95.0 \\
Added cream & 0 & 0 & 0 \\
Nonfat dry milk & 0 & 0 & 0 \\
Milk protein concentrate & 0 & 0 & 0 \\
On-site ultrafiltered milk & 0 & 0 & 0 \\
Skimmed cream & 0 & 0 & 0 \\
Fat in whey stream & 0.25 & 0.25 & 0 \\
Casein in whey stream & 0.10 & 0.10 & 0.25 \\
Whey protein in whey stream & 0.63 & 0.63 & 0.10 \\
Lactose in whey stream & 4.2456 & 4.2462 & 0.63 \\
Other solids in whey stream & 0.72 & 0.72 & 4.2462 \\
Water in whey stream & 79.0764 & 79.0755 & 0.72 \\
\hline
\end{tabular}

approximate mass balance equations, The second column is the solution to the approximate LP model with the exact composition of the whey stream recovered from Equations 7 and 8, and the third column is the solution to the NLP model. A comparison between the first 2 columns shows there is little difference in the whey stream compositions from the approximate mass balance equations compared with the exact. This was expected given the limited range of feasible serum solids for the cheese milk. Comparing the second and third columns shows there is no difference at all between the NLP solution and the approximate LP solution with exact mass balance equations. This example suggests that the simplest approach to solving the cheese NLP model may be to assume a composition for the serum solids in the cheese milk, solve the approximate LP model, and then recover the exact whey stream composition from Equations 7 and 8. This approach is used in the next example.

\section{Example 2}

The purpose of this example is to illustrate the net cost and yield implications of standardizing Cheddar cheese with ultrafiltered milk protein products. I solve the approximate LP model for 3 different scenarios: 1) ultrafiltered milk proteins are prohibited, 2) on-site ultrafiltered milk is permitted, and 3) both on-site ultrafiltered milk and milk protein concentrate are permitted. All 3 solutions are solved using the approximate LP model and recovering the exact whey stream from Equations 7 and 8. For completeness, they were checked against the solutions from the NLP model and found to be identical. The solutions to these 3 scenarios are provided in Table 4. 
Table 4. A comparison of standardization with ultrafiltered milk (UF) and milk protein concentrate (MPC)

\begin{tabular}{lccc}
\hline Variable & $\begin{array}{c}\text { No UF and } \\
\text { no MPC }\end{array}$ & $\begin{array}{l}\text { UF, } \\
\text { no MPC }\end{array}$ & $\begin{array}{c}\text { UF } \\
\text { and MPC }\end{array}$ \\
\hline Whole milk, kg & 95.0 & 64.0 & 68.8 \\
Skim milk, kg & 0 & 0 & 0 \\
Added cream, kg & 0 & 2.84 & 2.39 \\
Nonfat dry milk, kg & 0 & 0 & 0 \\
Milk protein concentrate, kg & 0 & 0 & 1.17 \\
On-site UF, kg & 0 & 5.03 & 0 \\
Skimmed cream, kg & 0 & 0 & 0 \\
Fat in whey stream, kg & 0.25 & 0.25 & 0.25 \\
Casein in whey stream, kg & 0.10 & 0.10 & 0.10 \\
Whey protein in whey stream, kg & 0.632 & 0.625 & 0.626 \\
Lactose in whey stream, kg & 4.246 & 3.251 & 3.322 \\
Other solids in whey stream, kg & 0.723 & 0.604 & 0.615 \\
Water in whey stream, kg & 79.076 & 57.015 & 57.483 \\
Yield & 0.105 & 0.139 & 0.138 \\
Input cost & $\$ 22.63$ & $\$ 23.46$ & $\$ 23.35$ \\
Vat costs & $\$ 3.56$ & $\$ 2.69$ & $\$ 2.71$ \\
Cream revenue & $\$ 0$ & $\$ 0$ & $\$ 0$ \\
Less whey revenue & $\$ 2.05$ & $\$ 2.05$ & $\$ 2.05$ \\
Net cost & $\$ 24.14$ & $\$ 24.10$ & $\$ 24.01$ \\
\hline
\end{tabular}

Table 4 shows a significant increase in cheese yield from the use of either ultrafiltered milk or milk protein concentrate. The use of ultrafiltered milk protein products increases the yield of Cheddar cheese from 0.105 to about 0.138 . In a $100-\mathrm{kg}$ vat, this is an increase of about $3.5 \mathrm{~kg}$ of cheese per batch.

The solutions in Table 4 also show where the cost reductions originate when ultrafiltered milk protein products are used: input costs actually rise under the use of either ultrafiltered milk or milk protein concentrate, but not enough to offset the reduced vat costs. The result is a reduction in the net cost for the $10 \mathrm{~kg}$ of Cheddar cheese from $\$ 24.14$ to $\$ 24.10$ with ultrafiltered milk and then to $\$ 24.01$ with milk protein concentrate. Naturally, these solutions are particular to the prices and vat costs used in this example and would be expected to change under different pricing assumptions.

A sensitivity analysis may be used to evaluate the true value of inputs not used in the model. These analyses are generated upon request in Excel and are printed into new worksheets. Here, I will demonstrate the use of a sensitivity analysis to calculate the value of NDM for use in standardization. The relevant results from a sensitivity analysis of the solution with ultrafiltered milk and milk protein concentrate are provided in Table 5 .

Table 5 gives to the "Allowable Increases" and "Allowable Decreases" of the variables in the problem, which are terms taken from the sensitivity analysis generated by the solver. The allowable increases and decreases are amounts that may be added to or subtracted from the coefficients in the objective function, respectively, without changing the value of the choice variables in the solution (Ragsdale, 2001). In this case, the objective function is the net cost function, and the coefficients are the prices. The set of nonzero variables in the solution is called the basis; zero-valued variables in the solution are not in the basis. Subtracting the allowable decrease from the price associated with a variable not in the basis gives the value at which that variable enters the basis. For instance, skim milk was not used in Cheddar cheese production at the price of $\$ 0.159 / \mathrm{kg}$, but would have been used at $\$ 0.148$ or less. Applying the method to NDM shows that it was too expensive at $\$ 1.985 / \mathrm{kg}$, but would have been competitive at $\$ 1.813$. Thus, with a working knowledge of sensitivity analyses, the model provides an easy method for pricing alternative inputs for use in cheese standardization.

\section{CONCLUSIONS}

I began with a conceptual model that expressed dairy products in terms of a set of 6 mutually exclusive and exhaustive components. The particular set used here, $\bar{L}$, was the smallest set necessary to express the constraints considered relevant, but it is not meant to be authoritative. If, for instance, a cheese maker wanted to separate nonprotein nitrogen from the other solids component, then he could do so in a straightforward manner by adding an element, say $n p n$, to the sets $L$ and $\bar{L}$. This would create a new whey stream variable, $w_{l}$, but the constraints would remain essentially unchanged. The conceptual model of dairy products, thus, provides an adaptable framework for the nonlinear cheese-making model and its linear approximation.

In a similar manner, the set of inputs used in cheese manufacture, $K_{x}$, may be expanded without affecting the underlying problem. If a cheese maker wanted to 
Table 5. Sensitivity analysis

\begin{tabular}{|c|c|c|c|c|c|}
\hline Variable & $\begin{array}{l}\text { Solution, } \\
\mathrm{kg}\end{array}$ & Price & $\begin{array}{l}\text { Allowable } \\
\text { increase }\end{array}$ & $\begin{array}{l}\text { Allowable } \\
\text { decrease }\end{array}$ & Value \\
\hline & & & 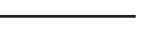 & & - \\
\hline Whole milk & 68.8 & $\$ 0.238$ & $\$ 0.010$ & $\$ 0.005$ & $\$ 0.238$ \\
\hline Skim milk & 0 & $\$ 0.159$ & $\inf ^{1}$ & $\$ 0.011$ & $\$ 0.148$ \\
\hline Added cream & 2.4 & $\$ 1.140$ & $\$ 0.055$ & $\$ 0.022$ & $\$ 1.140$ \\
\hline Nonfat dry milk & 0 & $\$ 1.985$ & inf & $\$ 0.171$ & $\$ 1.813$ \\
\hline Milk protein concentrate & 1.2 & $\$ 3.616$ & $\$ 0.081$ & $\$ 60.73$ & $\$ 3.616$ \\
\hline On-site ultrafiltered milk & 0 & $\$ 0.992$ & inf & $\$ 0.019$ & $\$ 0.973$ \\
\hline
\end{tabular}

${ }^{1}$ inf $=$ Infinite. Any increase in the associated price leaves the solution unchanged.

consider using condensed skim milk in his process, then he would add an element to the set $K_{x}$, call it $c s m$. He would then need to know the value of each component of the condensed skim milk, $\lambda_{c \mathrm{sm}, 1}$, and these values would enter the equations exactly as the other inputs. Other than these trivial changes, the models would be unaffected. Although some may find the expression of the models initially cumbersome, the symbolic language used here gives these models a flexibility they would otherwise lack.

I suggested two different approaches to solving the nonlinear problem faced by the cheese maker. In the first approach, I specified an algorithm to vary the serum solids content of the cheese milk, solving the consistent LP model at each point. The solution to the nonlinear problem was equal to the minimum net cost choice of serum solids content plus the associated solution to the consistent LP model. The second approach was to simply solve the approximate LP and recover the exact whey stream composition from the solution using Equations 7 and 8 . The results of Example 1 suggest that the 2 approaches may be equivalent in ordinary circumstances.

Example 2 shows the differences between standardization techniques with and without ultrafiltered milk protein products. The results show a significant increase in cheese yield with the use of ultrafiltered milk protein products. The yield without these products was $10.5 \mathrm{~kg}$ of cheese/hundredweight of cheese milk, and this increased to 13.9 and 13.8 with the use of ultrafiltered milk or milk protein concentrate, respectively. Ultrafiltered milk protein products also reduced the net cost per pound of cheese by $\$ 0.004 / \mathrm{kg}$ with the use of on-site ultrafiltered milk and by $\$ 0.013$ with imported milk protein concentrate.

Example 2 also illustrates an important point: yield maximization is not the same as net cost minimization. In the third scenario, on-site ultrafiltered milk and imported milk protein concentrate are both permitted for use in standardization, meaning that the solution from the second scenario is feasible in the third. A comparison between the second and third scenarios shows that it is possible for the lowest cost to be at a point where the yield is not maximized. Yield would have been higher with the use of on-site ultrafiltered milk, but the cost savings from the use of imported milk protein concentrate as a source of protein were so great that they offset the increased vat costs associated with the lower yield.

A comparison of the second and third scenarios in Example 2 suggests that the majority of cost reductions from the use of imported milk protein concentrate compared with ultrafiltered milk do not come from differences in yield, as the yields with the use of either were nearly identical. That is to say, with respect to this model, milk protein concentrate does not hold any significant functional advantage relative to ultrafiltered milk in increasing yield. Rather, the cost reductions from the use of milk protein concentrate are largely due to the fact that imported milk proteins are generally less expensive than those in domestically sourced ultrafiltered milk. If ultrafiltered milk could be supplied at a price of $\$ 0.973 / \mathrm{kg}$, as opposed to the $\$ 0.992$ price used in the model, the sensitivity analysis from the second example indicates that domestically sourced ultrafiltered milk would have been preferred to imported milk protein concentrate.

In developing these models, I endeavored to capture the assumptions and constraints commonly used in cheese manufacture. Expressing these constraints on a component basis lends clarity and flexibility to the model, and the linear approximation provides for an easy implementation of the model in any standard spreadsheet software package. It is my hope that this model will be useful to industry professionals for their planning and procurement decisions as well as to dairy scientists as a framework for further research.

\section{ACKNOWLEDGMENTS}

Funding for this research was provided by AGRICREE and a Hatch grant from USDA. I would also like to acknowledge the help of Mark Johnson from 
the Center for Dairy Research at the University of Wisconsin-Madison for his help in developing this model.

\section{REFERENCES}

Barcenas, C. 1999. Spreadsheet Strategies for Optimal Management of a Dairy Plant. Ph.D. Diss., Univ. Wisconsin, Madison.

Kerrigan, G. L., and J. P. Norback. 1986. Linear programming in the allocation of milk resources for cheese making. J. Dairy Sci. 69:1432-1440.

Papadatos, A., A. M. Berger, J. E. Pratt, and D. M. Barbano. 2002. A nonlinear programming optimization model to maximize net revenue in cheese manufacture. J. Dairy Sci. 85:2768-2785.
Papadatos, A., M. Neocleous, A. M. Berger, and D. M. Barbano. 2003. Economic feasibility of microfiltration of milk prior to cheesemaking. J. Dairy Sci. 86:1564-1577.

Ragsdale, C. T. 2001. Spreadsheet Modeling and Decision Analysis, 3rd ed. South-Western College Publishing, Cincinnati, OH.

Silberberg, E., and W. Suen. 2001. The Structure of Economics: A Mathematical Analysis, 3rd ed. Irwin/McGraw-Hill, New York, NY.

United States Department of Agriculture. 2003. Federal Milk Order Market Statistics, 2002 Annual Summary. Agricultural Marketing Services, Washington, DC.

United States International Trade Commission (USITC). 2004. Conditions of Competition for Milk Protein Products in the U.S. Market. USITC Pub. No. 3692. USITC, Washington, DC. 